\title{
O PROCESSO DA ASSISTÊNCIA AO PACIENTE COM LESÃO MEDULAR: GERENCIAMENTO DE CASO COMO ESTRATEGIA DE ORGANIZAÇÃO DA ALTA HOSPITALAR
}

\author{
PROCESS OF CARING FOR PATIENTS WITH SPINAL-CORD INJURY: CASE \\ MANAGEMENT AS A STRATEGY OF HOSPITAL DISCHARGE
}

Roxana I. Cardozo-Gonzales ${ }^{1}$, Tereza C. S. Villa² \& Maria H. L. Caliri ${ }^{3}$

\begin{abstract}
${ }^{1}$ Mestra em Enfermagem - Saúde Pública, ${ }^{2}$ Docente do Departamento de Enfermagem Materno-Infantil e Saúde Pública, ${ }^{3}$ Docente do Departamento de Enfermagem Geral e Especializada - Área Fundamental. Escola de Enfermagem de Ribeirão Preto da Universidade de São Paulo - Brasil.

Correspondência: Profa.Dra. Tereza Cristina Scatena Villa - Departamento Materno Infantil e Saúde Pública da EERP - USP. Av. Bandeirantes 3900 Campus universitário CEP: 14040-902 - Ribeirão Preto - SP. roxana@glete.eerp.usp.br
\end{abstract}

CARDOZO-GONZALES RI, VILLA TCS \& CALIRI MHL. O processo da assistência ao paciente com lesão medular: gerenciamento de caso como estrategia de organização da alta hospitalar. Medicina, Ribeirão Preto, 34: 325-333, jul./dez. 2001.

RESUMO: Este estudo tem como objeto conhecer como se realiza a assistência ao paciente com lesão medular, segundo a percepção da equipe de saúde. O eixo norteador do trabalho é compreender o processo da alta hospitalar do paciente com lesão medular e trazer, como proposta de organização, o Gerenciamento de Caso, que é uma modalidade de prestação de serviço, responsabilizando um profissional pelo total cuidado de saúde do paciente. Através de uma abordagem qualitativa, o estudo foi desenvolvido na Sala de Trauma e Urgências, Clínica Cirúrgica e Ortopedia de um hospital de nível terciário. Foi utilizada a entrevista semi-estruturada como técnica de coleta de dados. Participaram do estudo três médicos, um fisioterapeuta, dois assistentes sociais, um nutricionista, cinco enfermeiros, dois técnicos e três auxiliares de enfermagem que prestam assistência ao paciente com lesão medular. Os dados foram coletados durante os meses de abril e maio de 2000 e analisados pelo método de análise de conteúdo, através da análise temática. Identificaram-se como unidades temáticas, caraterização da assistência ao paciente com lesão medular durante a internação; necessidades ou problemas do paciente com lesão medular; problemas ou dificuldades na assistência ao paciente com lesão medular e preparo da alta: o real e o ideal.

UNITERMOS: Gerenciamento da Doença. Lesão da Medula Espinhal. Alta do Paciente.

\section{INTRODUÇÃO}

O número de portadores de lesão medular é um fato alarmante no mundo atual. Segundo More e Mandell $^{(1)}$, nos EUA, é calculado que existam 250.000 pessoas com danos de medula espinal, com média de 11.000 danos novos por ano, dos quais $55 \%$ são paraplégicos e $44 \%$ são tetraplégicos. Acidentes cau- sados por veículo motorizado respondem por $40 \%$ dos casos. Uma porcentagem de $25 \%$ é resultado de violência; $21 \%$ vêm de quedas; $10 \%$ vêm de acidentes por mergulho; $4 \%$ são resultados de acidentes de trabalho ou relacionados ao esporte. Em relação à faixa etária, $60 \%$ acontecem entre as idades de 16 e 30 anos, sendo mais frequientes na idade de 19 anos, e $60 \%$ são do sexo masculino. 
No Brasil, na maioria dos casos, tais lesões têm origem traumática, sendo o ferimento ocasionado por arma de fogo (FAF), acidente automobilístico e quedas, as causas externas mais frequientes. As vítimas desses traumatismos são predominantemente adultos jovens, com idade variando entre 18 e 35 anos e na proporção de quatro homens para uma mulher ${ }^{(2,3,4)}$.

$\mathrm{Na}$ literatura em Enfermagem, $\operatorname{Santos}^{(3)}{ }^{\left(\text {Faro }^{(5)}\right.}$ e Morooka ${ }^{(6)}$ identificaram problemas do paciente, durante a hospitalização e após a alta, tais como problemas de eliminação urinária, mobilização e alimentação. As autoras referem que o paciente com lesão de medula pode apresentar complicações digestivas, urinárias, deformidades, úlceras de pressão, trombose venosa profunda e disfunção autonômica.

Diante disso, o paciente com lesão medular é um tipo de paciente que, pela complexidade, demanda assistência específica e equipe interdisciplinar, atuando nas diferentes dimensões: social, psicológica e física, assegurando o cuidado holístico do paciente e a continuidade da assistência.

\subsection{Alta hospitalar}

Enfoques recentes têm considerado a alta hospitalar como um processo de transição do paciente, de um ambiente para outro, que pode ser o próprio domicílio ou qualquer outra instituição ${ }^{(7)}$.

Dentro das instituições de cuidado de saúde, o termo transição descreve movimento através dos níveis de cuidado, particularmente na transição do hospital para o domicílio ${ }^{(8)}$. Esse processo de transição inclui a transferência da responsabilidade do cuidado à família ou a outros profissionais de saúde. Isso tem se tornado uma grande preocupação entre os profissionais de saúde ${ }^{(7)}$.

Considera-se importante, neste estudo, abordar a questão da alta hospitalar dos pacientes (principalmente aqueles com problemas crônicos) que precisam de cuidado de saúde em casa, depois da hospitalização. Nesse sentido, a alta torna-se importante, pois não só afeta diretamente o paciente e a família, mas, também, os custos do sistema, já que se sabe que grande número de pacientes retornam ao hospital com recidivas que poderiam ter sido evitadas ou controladas no domicílio ou comunidade.

O preparo qualitativo da alta reflete-se num melhor seguimento de caso, assegurando maior integração do paciente à família e à comunidade e êxito no tratamento.

Nesse sentido, considera-se que, pensar e estudar a alta hospitalar, principalmente a do paciente com lesão medular, passa a ser fundamental, por considerá-la uma ação essencial, além da problemática, em relação ao planejamento e, também, às condições em que atualmente se realiza, quanto aos aspectos humano, técnico e financeiro, e à complexidade do problema de saúde.

Diante da situação exposta, acredita-se na importância de repensar novas estratégias de assistência e de implantar novas metodologias, visando ao atendimento integral ao paciente, assegurando os serviços necessários e a continuidade do cuidado, principalmente para os pacientes que necessitam dele após a alta hospitalar, de maneira eficiente, com o apoio da equipe multidisciplinar e boa relação custo-beneficio.

$\mathrm{Na}$ busca de alternativas, o gerenciamento de caso surge como uma estratégia-chave, tanto pela visão da melhoria dos resultados quanto pelo aspecto financeiro. O conceito e propósito do gerenciamento de caso são altamente congruentes para se alcançarem as metas individuais do paciente com lesão medular, relacionadas na melhora máxima da habilidade funcional e da qualidade de vida.

\subsection{Gerenciamento de caso}

Gerenciamento de caso é um processo sistemático de assistência ao paciente a partir de um trabalho interdisciplinar, iniciado na admissão do paciente e que nclui avaliação, implementação de planos de cuidado, coordenação e monitoração das opções de serviços, para que sejam alcançadas as necessidades de saúde individuais do paciente, através da comunicação entre os profissionais envolvidos na assistência e os recursos existentes dentro e fora da instituição de saúde ${ }^{(9)}$. Requer troca de tarefas específicas entre todos os membros da equipe de cuidado de saúde ${ }^{(10)}$.

Esse modelo apresenta um denominador comum: requer um único profissional de cuidado de saúde, seja um assistente social, médico ou enfermeiro. Como gerente, administra e responde pelo cuidado de saúde total de um grupo determinado de pacientes ${ }^{(11)}$. Provê intervenção precoce e apoio crescente aos indivíduos doentes, aumentando a qualidade do cuidado e também a qualidade de vida.

Diante do exposto, o presente estudo teve como objetivo geral, compreender o contexto da assistência do paciente com lesão medular no processo da alta hospitalar e, como objetivos específicos: 1) caraterizar a equipe de saúde, responsável pela assistência ao paciente com lesão medular $e$ o tipo de assistência prestada segundo o enfoque do gerenciamento de caso, 2) identifi- 
car as necessidades ou problemas do paciente com lesão medular no processo da alta hospitalar.

\section{METODOLOGIA}

Optou-se pela pesquisa qualitativa, definida por Minayo $^{(12)}$ como a pesquisa que trabalha com o universo de significados, motivos, aspirações, crenças, valores e atitudes, o que corresponde a um espaço mais profundo das relações, dos processos e dos fenômenos que não podem ser reduzidos à operacionalização de variáveis.

Segundo Lüdke \& André(13), a pesquisa qualitativa envolve a obtenção de dados descritivos, obtidos no contato direto do pesquisador com a situação estudada, enfatiza mais o processo que o produto e preocupa-se em retratar a perspectiva dos participantes.

Como local de investigação, foram eleitas três unidades de internação da Unidade de Emergência do Hospital das Clínicas, que prestam assistência ao paciente com lesão medular: Trauma e Urgências, Clínica Cirúrgica e Ortopedia. Realizou-se visita de observação, o que permitiu a obtenção dos dados iniciais e gerais do local de estudo e a identificação dos possíveis participantes para a realização da entrevista.

Optamos, neste estudo, por compreender o contexto da assistência ao lesado medular, atendido somente na Unidade de Emergência, e assim, nos restingimos ao processo assistencial ao paciente atendido pela equipe médica da Neurocirurgia. Destacamos que a equipe médica, a de enfermagem, e a do atendimento de Fisioterapia, Nutrição e Serviço Social, na instituição em estudo, referem-se tanto ao paciente da Ortopedia quanto ao da Neurocirurgia.

Participaram 17 trabalhadores de saúde que realizam assistência ao paciente com lesão medular por politraumatismo e na condição de paraplégico ou tetraplégico: três médicos, um fisioterapeuta, dois assistentes sociais, um nutricionista, cinco enfermeiros, dois técnicos de enfermagem, três auxiliares de enfermagem.

Considerou-se, como critério de inclusão, pelo menos, um trabalhador de saúde por unidade e por profissão. Elegeu-se a entrevista semi-estruturada como instrumento de coleta de dados. Elaborou-se um roteiro de entrevista para todos os profissionais, embora algumas perguntas tivessem sido mais direcionadas a determinada categoria profissional. A coleta de dados ocorreu durante o período de abril a maio de 2000, após prévio agendamento de horário.
Optou-se pela análise temática, uma das técnicas de análise de conteúdo a qual busca descobrir os núcleos de sentido que compõem uma comunicação cuja presença ou freqüência signifiquem alguma coisa para o objetivo analítico visado (...) a análise temática se encaminha para a contagem de freqüência das unidades de significação como definitórias do caráter do discurso ${ }^{(14)}$.

Operacionalmente, seguiram-se os passos preconizados por Minayo ${ }^{(14)}$, que abrangem as seguintes fases: pré-analise, exploração do material, tratamento dos resultados obtidos e interpretação.

Na primeira etapa, que constitui a fase de organização sistemática da pesquisa, foi realizado um levantamento bibliográfico, quando buscamos estudos relacionados ao planejamento da alta hospitalar. Além disso, foram procurados estudos sobre o referencial teórico a ser utilizado no confronto dos dados com a literatura, que é o gerenciamento de caso. É importante mencionar que o idioma predominante, na literatura encontrada foi o Inglês.

Numa segunda etapa, foram realizadas as entrevistas, as quais foram gravadas e transcritas na íntegra. A organização dos dados foi realizada através da leitura exaustiva e do processo de aprofundamento horizontal e vertical das mesmas. Foram destacadas as unidades de registro, agrupadas nos seguintes núcleos de sentido: caracterização da equipe de saúde que presta assistência ao paciente com lesão medular; organização do trabalho da equipe na assistência ao paciente com lesão medular; aspecto emocional; aspecto funcional; o preparo da alta.

Em seguida, os dados foram reagrupados para efeito de análise e interpretação, conformando-se, logo, as unidades temáticas deste estudo: caraterização da assistência ao paciente com lesão medular durante a internação; necessidades ou problemas do paciente com lesão medular; problemas ou dificuldades na assistência ao paciente com lesão medular; o preparo da alta: o real e o ideal. Posteriormente, numa terceira etapa, ocorreu a análise dos dados propriamente dita.

\section{ANÁLISE E DISCUSSÃO DE DADOS}

\subsection{Caraterização da assistência ao paciente com lesão medular durante a internação}

Equipe de saúde que presta assistência ao paciente com lesão medular. Através dos depoimentos dos entrevistados, identificou-se uma equipe de 
saúde constituída por profissionais médicos, de enfermagem, fisioterapeutas, nutricionistas e assistentes sociais. A equipe médica e de enfermagem realiza o atendimento de urgência e de emergência ao paciente politraumatizado, que ingressa na instituição após o trauma.

Os Médicos identificados na equipe foram: neurologista, ortopedista e neurocirurgião. Em relação aos pacientes atendidos pela equipe de Neurocirurgia, após a identificação do déficit neurológico do paciente, o neurocirugião assume a responsabilidade de conduzir o caso durante o período de internação do paciente. No processo da alta, ele realiza encaminhamento aos diferentes serviços, para complementação da assistência ao paciente.

A Equipe de Enfermagem é constituída pelo enfermeiro, técnico e auxiliar de enfermagem, sendo responsável pelo atendimento de enfermagem ao paciente com lesão medular durante 24 horas do dia e em todo o período de hospitalização. Ressaltam-se as atividades cotidianas, realizadas por essa equipe, no cuidado do paciente com lesão medular, como: alimentação, eliminação, higiene corporal, conforto físico, mudança de decúbito, bem como o trabalho educativo com o paciente e a família. É importante destacar que o trabalho do profissional de enfermagem não se limita apenas aos aspectos técnicos, mas, sim, às ações concretas, adotadas na solução de problemas do paciente.

O Assistente social realiza o atendimento ao paciente com lesão medular em duas etapas: na primeira etapa, trabalha a parte da identificação do paciente durante o período de admissão, em que o acidentado fica na sala de trauma. Numa segunda etapa, o assistente social participa ativamente de todo o processo de internação do paciente, principalmente no momento da alta, preocupando-se com a situação socioeconômica do paciente e da família.

O Fisioterapeuta é o profissional que está muito presente no processo da assistência ao paciente com lesão medular, desenvolvendo uma rotina de exercícios respiratórios e motores. Orienta o paciente e encaminha-o aos serviços a fim de dar continuidade ao cuidado e à reabilitação.

Em relação ao Nutricionista, existem dois profissionais da área, responsáveis pela elaboração, preparo e acompanhamento da alimentação de todos os pacientes da instituição. No processo da alta, o nutricionista realiza orientação ao paciente e família em relação à hidratação e higiene básica e à confecção e administração dos alimentos no domicílio.

Quanto à organização do trabalho da equipe na assistência ao paciente com lesão medular, os depoimentos mostraram uma equipe de saúde que presta assistência padronizada, em termos de rotinas hospitalares para o paciente com lesão medular, sem um plano de cuidados específico e elaborado conjuntamente. Cada trabalhador de saúde realiza suas funções restritas a sua área de trabalho.

“...até agora o que é conhecido em termo de trabalho na unidade de emergência é um trabalho completamente separado das outras especialidades a enfermagem faz o trabalho dela, o médico o dele, o serviço social"... (Entrevista $n^{o} 1$ ) (Assistente Social).

Diante da forma de organização do trabalho apresentado, nos depoimentos, pelos trabalhadores de saúde, observa-se a necessidade de reorganizar a assistência ao paciente com lesão medular com a participação da equipe de saúde, para atender às necessidades específicas do paciente.

\subsection{Necessidades ou problemas do paciente com lesão medular}

Desde o momento da admissão, na instituição de saúde, o paciente está ansioso, sendo sua principal preocupação o retorno da sensibilidade e a movimentação do corpo. Ele passa por diversas etapas, mostrando atitudes diferentes no processo de hospitalização. Alguns estão agressivos, não conversam, ou rejeitam a assistência do pessoal de saúde. Outros têm vergonha, ficam retraídos ou têm pensamento suicida.

“...o paciente chega com muita preocupação, ele te pergunta: vou andar? Não vou andar?..."(Entrevista $n^{o}$ 4) (Enfermagem).

É importante ressaltar, assim, que a mudança na vida do paciente, após o trauma da medula, não só afeta a ele como pessoa, mas também à família, que sofre uma mudança substancial em sua estrutura funcional.

Considerando o emocional do paciente com lesão medular - e o de sua família - como um aspecto crítico na assistência de saúde, é importante ressaltar a necessidade de trabalhar organizadamente (e em equipe) a forma de levar ao conhecimento do paciente e da família, o diagnóstico e a evolução do problema de saúde. No entanto, através das falas dos entrevis- 
tados, identifica-se que a informação para o paciente sobre o seu diagnóstico realiza-se de forma rápida, sem um mínimo de preparo do paciente e da família, situação que poderia estar danificando o emocional de ambos e dificultando a sua participação no processo.

“...geralmente tem o pessoal da equipe médica que fala para a família sobre a situação do paciente, quer dizer do diagnóstico, mas assim, de uma forma muito rápida e a família entra em desespero, eles falam tipo assim, ele tem uma lesão da medula a partir de agora ele não vai andar..." (Entrevista $n^{o}$ 4) (Enfermagem).

É importante refletir sobre o prejuízo emocional, ao paciente e à família, advindo dessa situação, o qual poderia ser evitado.

Quanto à autorização da alta hospitalar considera-se que, mesmo sendo um procedimento médico, ela deva ter a participação de outros profissionais da equipe, sendo importante observar e considerar outros critérios para que o planejamento e preparo da alta seja efetuado, abrangendo as condições fisiológicas, psicológicas, funcionais e sociais do paciente. Isso traz à tona a questão da integralidade da assistência na busca de um cuidado holístico ao paciente.

Diante do exposto, considera-se que a instituição ainda apresenta insuficiente organização em relação à assistência emocional do paciente, embora tal aspecto seja valorizado pelos informantes como um componente fundamental na assistência ao paciente com lesão medular deva ser considerado como prioridade.

“... a abordagem psicológica seria o suporte, é fundamental porque você compra o colchão ou a medicação, mas a parte psicológica não..."(Entrevista $n^{o}$ 1) (Assistente Social).

Quanto ao aspecto funcional, a complexidade da assistência ao paciente com lesão medular demanda não só maior tempo para a assistência,como também, a necessidade de um trabalho em equipe, além dos recursos materiais necessários ao atendimento.

Diante da complexidade das alterações físicas e emocionais, decorrentes de uma lesão de medula, é necessidade premente o planejamento de assistência específica para o paciente com esse tipo de problema. O planejamento deve incluir avaliação mensurada do estado do paciente, coordenação de serviços, monitoração das ações e resultados a fim de garantir a continuidade e a qualidade do cuidado.

\subsection{Problemas ou dificuldades na assistência ao paciente com lesão medular}

Os depoimentos mostram as dificuldades relacionadas à sobrecarga de trabalho dos trabalhadores de saúde e às restrições da infra-estrutura, relacionadas às condições do ambiente hospitalar, influenciando na qualidade da assistência ao paciente com lesão medular.

“...a Unidade de Emergência como HC são hospitais que têm muito volume cirúrgico de nossa área, então a gente não tem uma disponibilidade de $100 \%$, a hora que a família precisa da gente, às vezes estamos em cirurgia, outras vezes no ambulatório..."(Entrevista $n^{\circ}$ 16) (Medico).

A Unidade de Emergência do Hospital das Clínicas tem grande demanda de atendimento cirúrgico. Essa situação repercute no vínculo médico-família, assim como, também, nas formas de prestação de serviço em termos de equipe, em função da falta de tempo dos profissionais, principalmente do médico. Desse modo, é necessária a busca de soluções que permitam compartilhar responsabilidades de planejamento e coordenação da assistência durante o processo de hospitalização e, principalmente, no preparo da alta.

Quanto à rede de apoio social, ela é insuficiente em relação a alguns recursos básicos, como o respirador, já que muitos pacientes com lesão medular são dependentes do referido aparelho e a falta dele dificulta a alta do paciente, prolongando o tempo de hospitalização.

Assim, identificou-se a necessidade de uma instituição do tipo passagem, que ofereça condições de aprendizado quanto aos cuidados decorrentes das limitações após o trauma, para inicio da reabilitação imediata. Na maioria das vezes, após a alta do paciente com lesão medular, há um período de tempo variável para que ele se vincule a um programa de reabilitação ou simplesmente fique sujeito ao cuidado da família, no domicilio. Pelos depoimentos, percebe-se que isso acontece em virtude do número limitado de centros especializados, bem como devido a problemas de ordem social e econômica desses indivíduos. Para a reabilitação acontecer, é necessário contar com uma rede de suporte social, envolvendo organizações próprias, como associações públicas que priorizem um sistema de saúde eficiente e eficaz. 


\section{O PREPARO DA ALTA: o real e o ideal}

Os depoimentos enfatizam o problema da alta como a falta de acompanhamento e treinamento da família.

“...não é feito um treinamento prévio com a família, essa parte de orientação para casa é no momento da alta mesmo..." (Entrevista $\left.n^{\circ} 14\right)$ (Nutricionista).

Os depoimentos, abordam, também, a falta de maior entrosamento com órgãos públicos, recursos da comunidade, formais ou informais, como a assistente social da prefeitura, amigos, vizinhos ou voluntários de entidades religiosas.

“...acho que deveria ter um maior entrosamento com a comunidade, uma coisa assim, com a prefeitura, o serviço social da prefeitura, uma visita domiciliar, acho que deveria ser melhor trabalhado..."(Entrevista $\left.n^{o} 11\right)($ Enfermagem).

As falas apontam um outro aspecto, o relacionado ao horário de visita ao paciente. Essa política institucional afasta a família do ambiente hospitalar, embora o horário de visita seja o único meio de contato da família com o pessoal de saúde, para conversar e/ou esclarecer dúvidas. Esse horário, muitas vezes diverge do horário de trabalho dos profissionais de saúde, fragilizando o vínculo com a família, afastando-a do processo de cuidado.

“...o neurocirurgião não pode vir durante a visita, às vezes a própria família do paciente vem na hora errada e eles falam que não podem dar atenção...uma coisa bem afastada: o médico com a família."(Entrevista $\left.n^{o} 8\right)($ Enfermagem).

Charles et al. ${ }^{(15)}$, em estudos realizados no Canadá, apontam a comunicação entre paciente e médi$\mathrm{co}$, relacionada às necessidades e tratamento do paciente após a alta, como um componente crítico para o cuidado efetivo. A comunicação é um elemento-chave para que os responsáveis pela assistência possam tomar as decisões adequadas na resolução dos problemas identificados.

Para os participantes deste estudo, entretanto, a busca por uma melhora da assistência ao paciente com lesão medular é visualizada através de um trabalho em equipe. Explicam as vantagens desse tipo de trabalho para o paciente e para a própria instituição, já que evita a readmissão que representaria nova re- moção do lesado medular do ambiente domiciliar até o hospital, com alto custo para a instituição. Essa situação poderia ser evitada através do planejamento e preparo da alta em equipe.

Operacionalmente, a assistência ao paciente com lesão medular, na perspectiva de um trabalho em equipe, desenvolve-se a partir de discussão em grupo do estado de saúde do paciente, considerando-o como caso único, valorizando os critérios de cada profissional de saúde envolvido. Existe troca de informações das atividades que foram ou serão desenvolvidas pelos profissionais, um a um, durante todo o processo de hospitalização do paciente com lesão medular.

“...seria interessante que houvesse um grupo, uma equipe que discutisse sobre esses pacientes, de cada caso. $O$ médico apontando o aspecto clínico dele, como que está essa evolução, a enfermeira, no possível, mostrando o que ela vem fazendo, o que está acontecendo com o paciente, se tivesse psicóloga, vendo esse aspecto psicológico do paciente extremamente importante, nutrição cuidando do mais específico dessa parte alimentar, nutricional do paciente, aí teria uma troca de informação que enriqueceria todo mundo e principalmente beneficiaria o paciente. Não é você dividir conhecimento, muitas vezes, outros profissionais ficam meio arredios quando alguém vem chegando no espaço, mas não é dividir, é somar, se, por exemplo, o médico divide essa questão com outros profissionais, vai sobrar mais tempo para ele procurar informações na área dele, embora todo mundo tem que ter uma noção geral de tudo "(Entrevista $n^{\circ}$ 14) (Nutricionista).

Embora tenha sido identificada a valorização do preparo prévio da alta, o seguinte discurso mostra que a atuação dos profissionais de saúde (no preparo da alta) ocorre quando o paciente está saindo do hospital.

“...infelizmente a nossa atuação é principalmente mais no nível quando os pacientes estão indo de alta hospitalar, é bem no momento da alta..."(Entrevista $n^{\circ}$ 14)(Nutricionista).

Essa fala evidencia, mais uma vez, a falta de planejamento que vise satisfazer as necessidades não só do paciente como, também, da família, assim como a continuidade do cuidado. Desse modo, é fundamental considerar o planejamento da alta, como componente importante dentro do processo da assistência ao paciente com lesão medular pela equipe multiprofissional. 
Eis que os depoimentos identificam três elementos fundamentais além do próprio paciente no planejamento e preparo da alta do paciente: a família, a equipe de saúde e os órgãos públicos que, necessariamente, devem se manter interligados a fim de ser assegurada a continuidade do cuidado do paciente com lesão medular após a alta hospitalar.

Ressalta-se a importância de a família estar dispondo dos recursos materiais, de receber orientação e acompanhamento, principalmente nos primeiros dias após a alta, para verificar a incorporação das orientações no cuidado ao paciente, no domicílio. A família é um elemento importante para oferecer segurança ao paciente e à própria instituição na continuidade da assistência após a alta hospitalar.

“...importante chamar a família para participar dos cuidados e orientar. Os profissionais deveriam estar envolvidos nesse trabalho com a família, o trabalho teria que ser um trabalho maior" (Entrevista $\left.n^{o} 4\right)$ (Enfermagem).

Ressalta-se a visita domiciliar como uma atividade de apoio para a família estar realizando os cuidados, segundo o treinamento recebido no hospital.

\subsection{Gerenciamento de caso, como uma propos- ta de organização da assistência, no prepa- ro da alta ao paciente com lesão medular.}

Diante da atual forma de organização da assistência e preparo da alta do paciente com lesão medular, reafirma-se, mais uma vez, a necessidade de repensar a forma de organização da assistência a esse tipo de paciente, na perspectiva de um trabalho integral, que permita uma assistência holística e participação ativa da família ou cuidador. Para que esse tipo de assistência seja alcançado, é necessária atuação conjunta da equipe com eficiente articulação das ações.

Desse modo, coloca-se o gerenciamento de caso como estratégia de integração da equipe e das ações, durante a internação, e o processo de preparo da alta hospitalar, na busca de melhores resultados do atendimento ao paciente com lesão medular.

É importante assumir o desafio da interdisciplinaridade baseada numa perspectiva de diálogo e interação entre disciplinas, e nos dos conhecimentos justapostos em torno de um mesmo problema, onde cada qual trabalha dentro das fronteiras da disciplina para alcançar resultados independentemente ${ }^{(16)}$. No gerenciamento de caso, o gerente é, freqüentemente, o colaborador do cuidado ao paciente, no sentido de integrar a equipe para negociar o melhor plano de cuidado para o paciente. Canaliza informações para todas as outras partes envolvidas na assistência ao paciente ${ }^{(17)}$. Essa informação permite integrar experiências de profissionais de cuidado de saúde de várias disciplinas e reconcilia visões discrepantes, de forma a alcançar metas de cuidado para o paciente ${ }^{(18)}$.

Rorden \& Taft e Williams, citados por Pichitpornchai et al. ${ }^{(7)}$, referem que o planejamento da alta é um processo pelo qual o paciente, família e a equipe constituída por várias disciplinas colaboram para facilitar a transição do paciente para outro ambiente ou para uma próxima fase.

O planejamento da alta tornou-se, dessa forma, elemento essencial no ambiente hospitalar, na prestação da assistência, contribuindo para o progresso do paciente ao longo do contínuo ${ }^{(7)}$. Inclui avaliação da continuidade do cuidado da saúde, necessário fora do hospital, e o trabalho com o paciente e sua família para desenvolver um plano realístico a ser realizado após alta. Assim, o planejamento da alta identifica as necessidades potenciais na comunidade ou ajuda a fazer arranjos necessários para dar assistência para o paciente e família ${ }^{(19)}$.

Evidenciada a importância do planejamento da alta hospitalar, o gerenciamento de caso considera-o um componente fundamental ${ }^{(1)}$ para garantir ótimos resultados na assistência e após a alta hospitalar do paciente.

O gerenciamento de caso inicia o planejamento da alta, desde a admissão do paciente, através da avaliação total de elementos médicos, psicossociais e financeiros de cada caso. Considera cada caso como um aspecto único, que determina o planejamento dos serviços e a coordenação dos detalhes ${ }^{(17)}$. Durante o processo de hospitalização, o gerente de caso reúne o paciente para estabelecer concordância e colecionar informações relacionadas ao presente e ao passado médico, assunto psicossocial, assuntos ambientais e espirituais. Essas informações são usadas para identificar problemas (designando o curso do tratamento) e iniciar um plano de alta $^{(1)}$. Posteriormente, o gerente de caso negocia o plano com o paciente e os profissionais de saúde que provêem assistência, implementa e monitora o plano, analisando os resultados, intervindo e reajustando o plano, quando necessário ${ }^{(16)}$.

No contexto do planejamento da alta e do próprio processo de assistência ao paciente com lesão medular, o gerenciamento de caso identifica a família como um elemento fundamental. Powell ${ }^{(17)}$ ressal- 
ta que, para que o planejamento da alta tenha sucesso, o paciente e família devem participar ativamente de todas as decisões, incluindo quaisquer mudanças no plano de cuidado. Esse aspecto é trabalhado amplamente pelo gerente de caso durante o processo de hospitalização.

Acredita-se, então, que o gerenciamento de caso, pelas suas caraterísticas, possa contribuir para melhoria dos problemas identificados, não apenas os relativos às questões do preparo da alta hospitalar do lesado medular, mas, também, aqueles problemas que dizem respeito ao aspecto emocional, físico ou orgânico do paciente, visando ao cuidado holístico e à continuidade da assistência.

\section{CONSIDERAÇÕES FINAIS}

O paciente com lesão medular é um tipo de paciente que, pelas suas especificidades, defronta-se com grandes problemas no atual sistema de saúde devido a fatores socioeconômicos e políticos que determinam o modelo do cuidado.

Por outro lado, existe a grande necessidade da continuidade do cuidado ao paciente após a alta hospitalar, sendo necessária assistência e preparo da alta com qualidade, inserindo, nesse processo, a família, através da participação ativa no cuidado do paciente.

Este estudo mostrou que, em uma unidade de internação de um hospital-escola, a assistência ao paciente com lesão medular não se diferencia daquela dada aos pacientes das outras patologias. Em termos de estrutura física, o paciente, que carece de uma unidade específica, é tratado nas dependências como do ambiente hospitalar. Em relação à assistência, não ocorre o envolvimento de todas as disciplinas com um objetivo comum, cada profissional realiza suas funções restritas à sua área de trabalho. Não existe um plano de cuidados específicos, elaborado conjuntamente. Isso traz, como resultado, insuficiente articulação e integração das ações na assistência, com grande repercussão na continuidade do cuidado, principalmente após a alta. Quanto à rede de apoio social, ela foi identificada como insuficiente para responder às necessidades de uma assistência de nível complexo como é a devida ao paciente com lesão medular Assim, ressalta-se a falta de uma instituição de retaguarda a fim de garantir a reabilitação precoce e a continuidade do cuidado do paciente através do cumprimento das orientações pelos profissionais, família ou cuidador.

Considera-se relevante destacar a reabilitação como um aspecto de fundamental importância, na vida do paciente com lesão medular, para manter capacidade dele e evitar atrofia muscular. Além da melhora da capacidade motora, a reabilitação também eleva a auto-estima do paciente, favorecendo sua adaptação ao novo estilo de vida.

Tomando como referência o papel da família, na continuidade do cuidado do paciente com lesão medular, no domicílio, é relevante enfatizar a necessidade do treinamento da mesma - através do planejamento de atividades (envolvendo a equipe de saúde) durante o processo de internação do paciente - orientar, e mostrar, de forma mais organizada, os cuidados necessários com o paciente, esclarecer suas dúvidas e certificar-se da incorporação das orientações dadas a ela.

Diante do exposto, considera-se que o lesado medular é um tipo de paciente com particularidades específicas, que necessariamente demanda uma assistência que requer o gerenciamento de caso por um profissional qualificado, que coordene e advogue por ele e sua família durante todo o processo de internação, envolvendo a equipe de saúde e o processo da alta.

A prática dessa modalidade de prestação de serviço contribui para a melhora da assistência e o preparo da alta através de um planejamento que deve ser iniciado na admissão do paciente e continuado durante todo o processo de internação na instituição, avaliando todos os aspectos relacionados ao paciente, assim como os recursos (humanos e materiais) disponíveis e necessários, que permitam uma alta com sucesso, garantindo a continuidade do cuidado. $\mathrm{O}$ gerente de caso integra todas as partes envolvidas no cuidado do paciente, tais como a equipe de saúde, família (ou cuidador) e o próprio paciente. Assim, estabelece ligações a fim de obter um atendimento mais apropriado e ainda com custo efetivo para o paciente e para a instituição. O gerente de caso negocia o sistema de cuidado de maneira que beneficie todos os envolvidos no processo.

Nesse sentido, considera-se importante, em próximos estudos, aprofundar o conhecimento sobre tal modalidade de prestação de serviço e propor sua aplicação na assistência de pacientes específicos, crônicos ou de grande complexidade. Justifica-se, ainda mais, a necessidade de se estudar essa modalidade devido a possíveis utilidades de sua aplicação, adequadas à realidade dos serviços de saúde brasileiros, na tentativa de responder às necessidades emergentes na prestação de serviços. 
CARDOZO-GONZALES RI, VILLA TCS \& CALIRI MHL. Process of caring for patients with spinal-cord injury: case management as a strategy of hospital discharge. Medicina, Ribeirão Preto, 34: 325-333, july/dec. 2001.

ABSTRACT: This study aimed at understanding the process of discharge from hospital of patients with spinal-cord injury according to health professional teams. The research was conducted considering the process of discharge from hospital of patients with spinal-cord injury introducing case management, which is a modality of service that puts one professional in charge of the integral healthcare of patients. Through a qualitative analysis the study was conducted with health professionals from the Emergency Department, Surgery Clinic and Orthopedics Clinic of Ribeirão Preto Emergency Hospital (UE). Semi-structured interviews were used as a technique to collect data during April, 2000, with 3 physicians, 1 physical therapist, 2 social workers, 1 dietician, 5 nurses, 2 nursing technicans and 3 nursing auxiliaries. The thematic units of the research comprised: characterization of the care given to patients with spinal-cord injury during hospital stay; needs or problems of patients with spinal-cord injury; problems or difficulties in caring for patients with spinal-cord injury; preparation for discharge: what is real and what is ideal.

UNITERMOS: Disease Management. Spinal Cord Injury. Patient Discharge.

\section{REFERÊNCIAS BIBLIOGRÁFICAS}

1 - MORE PK \& MANDELL S. Nursing case management: an evolving practice. McGraw-Hill, New Jersey, 1997.

2 - SPOSITO MM; FILHO JL; BRAGA FM \& NOVO NF. Paraplegia por lesão medular: estudo epidemiológico em pacientes atendidos para reabilitação. Rev Paul Med 104: 196-202, 1986.

3 - SANTOS LCR. Lesão traumática da medula espinhal: estudo retrospectivo de pacientes internados no Instituto de Ortopedia e Traumatologia do Hospital das Clínicas da Faculdade de Medicina da Universidade de São Paulo entre 19821987. Dissertação de Mestrado, Escola de Enfermagem da USP, São Paulo. p. 1-102, 1989.

4 - FARO ACM. Estudo das alterações da função sexual em homens paraplégicos. Dissertação do Mestrado, Escola de Enfermagem da USP, São Paulo. p.1-98, 1991.

5 - FARO ACM. Do diagnóstico à conduta de enfermagem. Tese de Doutorado, Escola de Enfermagem da USP, São Paulo. p. 1-208, 1995.

6 - MOROÓKA M. Autocateterismo vesical intermitente técnica limpa descrição do procedimento realizado pelos pacientes com lesão medular. Dissertação de Mestrado, Escola de Enfermagem da USP, São Paulo p. 52, 2000.

7 - PICHITPORNCHAI W; STREETA \& BOONTONG T. Discharge planning and transitional care: issues in Thai nursing. Int $\mathbf{J}$ Nurs Stud 36: 355-362, 1999.

8 - FERREIRA AB. Novo Aurélio Século XIX: o dicionário da língua portuguesa. 3 ed, Nova Fronteira, Rio de Janeiro, 1999.

9 - McCOLLOM P \& SAGER D. Case management. In: HOEMAN SP. Rehabilitation nursing: process and application. 2 ed, MOSBY, St. Louis, p. 102-104,107, 1996.

10 - RAWSKY E. Building a case management model in a small community Hospital. Nurs Manag 27: 49-51, 1996.
11 - ROGERS M; RIORDAN J \& SWINDLE D. Community-based nursing case management pays off. Nurs Manag 22: 3034, 1991.

12 - MINAYO MC. O desafio do conhecimento: pesquisa qualitativa em saúde. 5 ed, Hucitec - Abrasco, São Paulo, 1998.

13 - LÜDKE M \& ANDRE MEDA. Pesquisa em Educação: abordagens qualitativas. EPU, São Paulo, 1986.

14 - MINAYO MCF. O desafio do conhecimento: pesquisa qualitativa em saúde. 2 ed, HUCITEC/ABRASCO, São Paulo, 1994.

15 - CHARLES C; CHARBERS L; OBRIENB; HAYNES RB \& LABELLE R. How was your hospital stay? Patients'reports about their care in Canadian hospitals. Can Med Assoc J 150:1813-1822,1994.

16 - MUMMA C M \& NELSON A. Models for theory-based practice of rehabilitation nursing. In: HOEMAN, S. P. Rehabilitation nursing: process and application, 4 ed, MOSBY, St. Louis, p. 21-33, 1996.

17 - POWELL SK. Nursing case management. Lippincott, New York, 1996.

18 - HANSEN H; BULL M \& GROSS C. Interdisciplinary collaboration and discharge planning communication for elders. $\mathbf{J}$ Nurs Adm 28: 37-46, 1998.

19 - LOWENSTEIN AJ \& HOFF PS. Discharge Planning: a study of nursing staff involvement. J Nurs Adm 24: 45-50, 1994.

Recebido para publicação em 20/03/2001.

Aprovado para publicação em 04/07/2001. 